\title{
Presentation of a Mullerian Duct Cyst in a Male Child with Prolonged Fever
}

\author{
Dong Hee Kang, Su Yeon Kim, Young Eun Kim, Sung Hwan Byun
}

Department of Pediatrics, Bundang Jesaeng General Hospital, Seongnam, Korea

\begin{abstract}
The Mullerian duct cyst (MDC) occurs due to incomplete focal regression and saccular dilatation of the paramesonephric duct. It is usually found asymptomatic in adults, but it also shows symptoms such as ejaculatory impairment, hematospermia, pelvic pain, urinary retention, and urinary tract infections. MDCs are rare in children. Transrectal ultrasonography, computed tomography, and magnetic resonance imaging are useful for diagnosis. Treatment is based on age, clinical symptoms as well as size and location of the cyst. Here, we report a case of a 19-month-old boy with an infectious MDC that presented with fever. He has improved with antibiotic treatment, not surgical resection.
\end{abstract}

Keywords: Mullerian duct cyst; Fever; Child

\section{INTRODUCTION}

The Mullerian duct cyst (MDC) is a rare pelvic cystic lesion developed from the persistent remnant of the Mullerian duct that should be normally regression by anti-Mullerian hormones early in gestation. A prevalence of $1 \%-5 \%$ has been reported for MDCs [1], but the symptomatic presentation is very rare. We report a case of the infection of the MDC in a 19-months-old boy presenting with fever. We could find a large circular cystic mass located in the midsection between the bladder and the rectum by computed tomography (CT) scan.

\section{CASE REPORT}

A 19-month-old male was admitted because of a fever that had persisted for 6 days. He had three to four loose stools during this period, without any other symptoms. His medical history was unremarkable. Vital signs at admission were normal, and only his body temperature was elevated to $40.0^{\circ} \mathrm{C}$. Physical examination revealed no abnormal findings. Initial laboratory findings have been provided in Table 1. Urine test results were normal. The patient was intravenously administered empirical antibiotics (ampi- cillin/sulbactam). However, after 5 days of antibiotic therapy, the fever (up to $38.8^{\circ} \mathrm{C}$ ) continued. On hospital day 5 , the mother reported the patient curling up suddenly with slight moaning and relaxing afterward. Next, abdominal ultrasonography (US) was performed. A cystic mass measuring approximately $4.5 \mathrm{~cm}$ surrounded by thick walls posterior to the bladder and anterior to the sacrum was found. Hyperechoic contents were visible inside the cyst, indicative of infection (Fig. 1A). Subsequently, abdominalpelvic CT was performed to determine the location, shape, and other characteristics of the cyst. The imaging results showed a 4.7 $\mathrm{cm}$ circular cystic mass located in the midsection between the bladder and the rectum (Fig. 1B, C). This cyst was diagnosed as an MDC because it was not connected to the bladder or urethra and was not accompanied by other anomalies. After a urology consultation, continuous conservative treatment was initiated before surgical treatment. Intravenous cefotaxime injection was added to the treatment. On hospital day 7, the fever subsided, and curling up was no longer observed. Blood tests conducted on hospital day 11 showed that inflammation levels had decreased to $14,200 / \mu \mathrm{L}$ of white blood cell count (51.7\% of segmented neutrophils) and C-reactive protein level was $0.51 \mathrm{mg} / \mathrm{dL}$. Twelve days after admission, he was discharged and administered cefpodoxime for 3 additional 
Table 1. Patient's laboratory findings and reference ranges

\begin{tabular}{lcc}
\hline Laboratory test & Patient values & Reference range \\
\hline Hemoglobin (g/dL) & 11.5 & $10.5-14.0$ \\
Platelet $(/ \mu \mathrm{L})$ & 343,000 & $150,000-400,000$ \\
White blood cell (/ML) & 13,500 & $6,000-15,000$ \\
Segmental neutrophil (\%) & 45.8 & $10.0-80.0$ \\
Lymphocyte (\%) & 46.3 & $20.0-50.0$ \\
Erythrocyte sedimentation rate (mm/hr) & 60 & $1-9$ \\
C-reactive protein (mg/dL) & 3.59 & $0.00-0.80$ \\
Procalcitonin (ug/L) & 1.07 & $0.00-0.49$ \\
Blood urea (mg/dL) & 11.3 & $8.0-22.0$ \\
Serum creatinine (mg/dL) & 0.29 & $0.80-1.50$ \\
Aspartate aminotransferase (IU/L) & 30 & $0-40$ \\
Alanine aminotransferase (IU/L) & 18 & $0-40$ \\
Blood culture & No growth & No growth
\end{tabular}

weeks. Three weeks after discharge, a follow-up abdominal US of the cyst showed that it had reduced in size $(1.8 \mathrm{~cm})$ and did not have any fluid or wall thickening (Fig. 1D). Subsequently, oral antibiotics were discontinued.

The patient's caregiver provided written informed consent for the publication of clinical details and images.

\section{DISCUSSION}

The Mullerian duct, which originates from the embryonic mesoderm, is a structure that forms during the embryonic period [2]. In early embryos, a pair of Mullerian and Wolffian ducts are formed regardless of sex. However, in the male embryo, the Mulle-
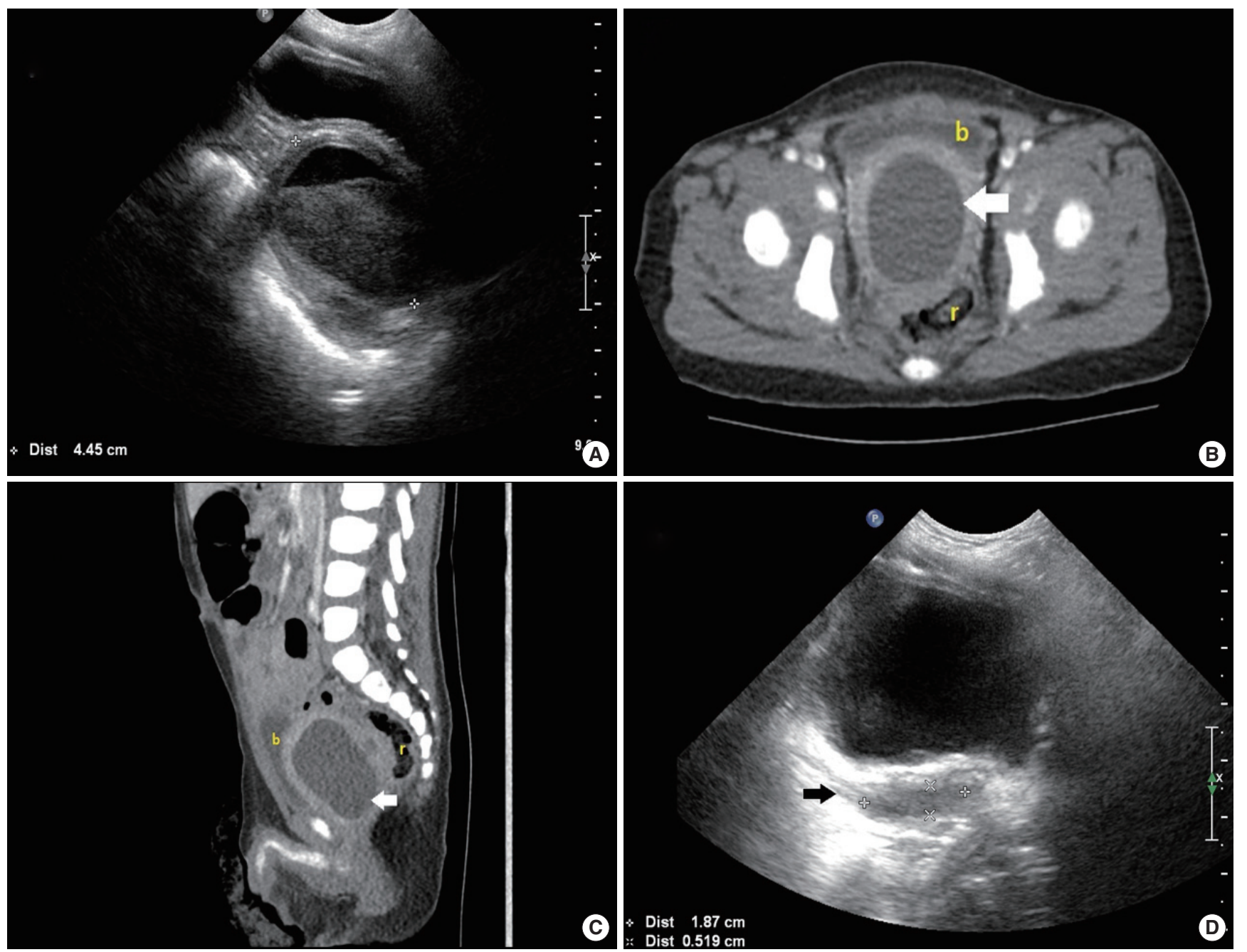

Fig. 1. (A) The transverse scan of the lower abdomen shows an approximately $4.5 \mathrm{~cm}$ thick-walled hyperechoic cyst in the presacral space. (B, C) Abdominal-pelvic computed tomography and CE show an approximately $4.7 \mathrm{~cm}$, thick-walled midline cystic lesion in the pelvis (white arrows). The cyst is round-shaped and located between the urinary bladder (b) and rectum (r). (D) The collapsed state (size reduction from 4.5 to $1.8 \mathrm{~cm}$ ) of the previous midline cystic lesion in the pelvis with improvement in peripheral wall thickening and internal echogenicity (black arrow). 
Kang DH, et al. • Presentation of a Mullerian Duct Cyst in a Male Child with Prolonged Fever

rian duct degenerates while the Wolffian duct differentiates into male reproductive structures such as the vas deferens, epididymis, and seminal vesicles [3]. Conversely, in female embryos, the Mullerian duct differentiates into the oviducts, uterus, and upper vagina, while the Wolffian duct degenerates [3]. In males, the antiMullerian hormone is secreted from the Sertoli cells in the testicles at around 7 to 10 weeks of gestation, mediating Mullerian duct regression [2,3]. MDCs are remnants of the Mullerian duct that dilate to form cysts.

MDCs are asymptomatic and usually found in people aged 20 to 40 years old [4]. However, symptoms often occur when an MDC presses against the lower urinary tract or the rectum, obstructing the ejaculatory duct. Symptoms include pelvic pain, urinary disorders, urinary tract infections, disturbance of ejaculation, hematospermia, and infertility. MDCs are rare, especially in children $[1,4,5]$.

The differential diagnosis of midline cysts in the pelvic cavity in males includes prostatic utricle cysts, prostatic retention cysts, prostatic abscesses, bladder diverticulum, rectal duplication cyst, or any pelvic neoplasm [2]. An MDC extends from the upper prostate and presents as circular or oval shapes, varying in size. It is not connected to the prostatic urethra, but rather to the verumontanum [6,7]. It is usually not accompanied by other urinary malformations and is rarely accompanied by ipsilateral renal agenesis [8]. Transrectal US, CT, and magnetic resonance imaging are useful for differential diagnosis.

A small, asymptomatic Mullerian cyst requires no intervention. Surgical treatment can be considered if the cyst is large or if there are obstructive symptoms [4]. Recently, surgical methods using laparoscopic or robot-assisted techniques for cyst removal have been reported [9]. If the cyst size is large, open surgical excision is the appropriate treatment [4]. However, An MDC is located in the deep location of the pelvis and adjacent to important surrounding structures such as bladder, rectum, urethra, vas deferens, pelvic nerves, ureter, and urethra, so extra caution is needed during the surgical treatment [10]. Complications such as impotence, lack of ejaculation, urinary tract infection, external sphincter injury, and rectourethral fistula have been reported in case of surgical opening and may result in prolonged hospitalization or recovery [11].

We report a case of an infectious MDC using a radiologic examination in a child. There have been just a few cases reported for MDC in children in the medical literature. Chinya et al. [5] have reported a case where they performed laparoscopic cyst excision on a 9-month-old child with an MDC experiencing urinary tract obstruction. In Korea, there is a case where they performed laparoscopic cystectomy on a 1-year-old boy with an epididymis infection caused by an infected MDC [12]. Unlike previously reported cases, conservative treatment, rather than surgical treatment, improved symptoms, and a radiologic follow-up examination confirmed that cyst infections improved. This prevented complications caused by surgery. In conclusion, MDC with symptoms is rare in children and imaging tests are useful for diagnosis and follow-up.

\section{CONFLICT OF INTEREST}

No potential conflict of interest relevant to this article was reported.

\section{REFERENCES}

1. Coppens L, Bonnet P, Andrianne R, de Leval J. Adult Mullerian duct or utricle cyst: clinical significance and therapeutic management of 65 cases. J Urol 2002;167:1740-4.

2. Unnikrishnan R, Herts BR, Sabanegh E. Symptomatic Mullerian duct cyst. J Urol 2012;188:963-4.

3. Mullen RD, Behringer RR. Molecular genetics of Mullerian duct formation, regression and differentiation. Sex Dev 2014;8:281-96.

4. Shebel HM, Farg HM, Kolokythas O, El-Diasty T. Cysts of the lower male genitourinary tract: embryologic and anatomic considerations and differential diagnosis. Radiographics 2013;33:1125-43.

5. Chinya A, Raj P, Sinha SK, Sarin YK. Symptomatic Mullerian duct cyst in a male infant. APSP J Case Rep 2016;7:31.

6. Parsons RB, Fisher AM, Bar-Chama N, Mitty HA. MR imaging in male infertility. Radiographics 1997;17:627-37.

7. McDermott VG, Meakem TJ 3rd, Stolpen AH, Schnall MD. Prostatic and periprostatic cysts: findings on MR imaging. AJR Am J Roentgenol 1995;164:123-7.

8. Devaraju S, Nerli RB, Hiremath MB. A large mullerian duct cyst presenting as an abdominal mass with ipsilateral renal agenesis: an unusual presentation. Nephrourol Mon 2012;4:640-3.

9. Hong YK, Onal B, Diamond DA, Retik AB, Cendron M, Nguyen HT. Robot-assisted laparoscopic excision of symptomatic retrovesical cysts in boys and young adults. J Urol 2011;186:2372-8.

10. Krstic ZD, Smoljanic Z, Micovic Z, Vukadinovic V, Sretenovic A, Varinac D. Surgical treatment of the Mullerian duct remnants. J Pediatr Surg 2001;36:870-6.

11. Desautel MG, Stock J, Hanna MK. Mullerian duct remnants: surgical management and fertility issues. J Urol 1999;162(3 Pt 2):1008-14.

12. Kim TH, Lee KS, Sung GT, Cho WY. Laparoscopic excision of Mullerian duct cyst in 1 year old boy. Korean J Pediatr Urol 2012;4:32-5. 